TRANSACTIONS OF THE

AMERICAN MATHEMATICAL SOCIETY

Volume 352, Number 8, Pages 3799-3820

S 0002-9947(00)02563-0

Article electronically published on March 27, 2000

\title{
SCATTERING MATRICES FOR THE QUANTUM $N$ BODY PROBLEM
}

\author{
ANDREW HASSELL
}

\begin{abstract}
Let $H$ be a generalized $N$ body Schrödinger operator with very short range potentials. Using Melrose's scattering calculus, it is shown that the free channel 'geometric' scattering matrix, defined via asymptotic expansions of generalized eigenfunctions of $H$, coincides (up to normalization) with the free channel 'analytic' scattering matrix defined via wave operators. Along the way, it is shown that the free channel generalized eigenfunctions of Herbst-Skibsted and Jensen-Kitada coincide with the plane waves constructed by Hassell and Vasy and if the potentials are very short range.
\end{abstract}

\section{INTRODUCTION}

Consider the Schrödinger operator $P=\Delta+V$, where $\Delta$ is the positive Laplacian on $\mathbb{R}^{D}$ and $V$ is a real valued function with decay conditions at infinity. A fundamental object in the analysis of $P$ is the scattering matrix. This may be looked at in (at least) two different ways, leading to two different definitions of the scattering matrix which will be called the analytic scattering matrix and geometric scattering matrix here. It is well known that the two definitions coincide up to normalization for two-body operators. The purpose of this paper is to show that they also coincide for a class of many-body operators.

Most rigorous work up to date on the $N$ body problem has analyzed the wave operators, very closely related to the analytic scattering matrix (see below). The wave operators are the natural tool for studying the question of asymptotic completeness and analyzing the structure of the spectrum. On the other hand, recently the geometric scattering matrix was shown to be amenable to a rather precise singularity analysis 3], [16]. It is not known how to obtain similar results in any direct way for the analytic scattering matrix. Therefore, it is a significant question whether these two scattering matrices coincide.

Let us first discuss the two definitions in the case of two-body operators. For the sake of exposition, let us assume for the moment that $V$ is 'very short range', that is, rapidly decreasing at infinity. The analytic scattering matrix may be thought of as encapsulating the difference between the long time behaviour of solutions $\psi(\cdot, t)=e^{-i t P} \psi$ to the time dependent Schrödinger equation $i \partial_{t} \psi=P \psi$, compared to the free evolution $e^{-i t P_{0}} \psi$ given by the free Hamiltonian $P_{0}=\Delta$. An initial state $\psi$ behaves asymptotically in the past as the free state with initial condition

Received by the editors February 11, 1998.

2000 Mathematics Subject Classification. Primary 35P25, 81U10, 81U20, 35S05.

Key words and phrases. $N$ body problem, scattering theory, scattering matrix, scattering calculus. 
$\psi_{\text {in }}$ if

$$
\left\|e^{-i t P} \psi-e^{-i t \Delta} \psi_{\text {in }}\right\|_{2} \rightarrow 0 \text { as } t \rightarrow-\infty,
$$

that is, if $\psi=\mathrm{s}-\lim _{t \rightarrow-\infty} e^{i t P} e^{-i t \Delta} \psi_{\mathrm{in}}$, and asymptotically in the future as the free state with initial condition $\psi_{\text {out }}$ if

$$
\left\|e^{-i t P} \psi-e^{-i t \Delta} \psi_{\text {out }}\right\|_{2} \rightarrow 0 \text { as } t \rightarrow \infty,
$$

that is, if $\psi=\mathrm{s}-\lim _{t \rightarrow \infty} e^{i t P} e^{-i t \Delta} \psi_{\text {out }}$. Thus, in the Heisenberg picture, the overall effect of the perturbation $V$ of the free operator is to transform $\psi_{\text {in }}$ to $\psi_{\text {out }}$. This is described by the scattering operator $S$, which by definition is

$$
\begin{gathered}
S=\left(\Omega^{-}\right)^{*} \Omega^{+}, \quad \Omega^{ \pm}=\mathrm{s}-\lim _{t \rightarrow \mp \infty} e^{i t P} e^{-i t \Delta} ; \\
S: \psi_{\text {in }} \mapsto \psi_{\text {out }} .
\end{gathered}
$$

The wave operators $\Omega^{ \pm}$are partial isometries on $L^{2}\left(\mathbb{R}^{D}\right)$ with range $H_{\text {ac }}(P)$, the continuous spectral subspace of $P$. The strong limits defining them exist under rather general conditions on $V$ [14].

It is more convenient to study this in Fourier space, so consider

$$
\mathcal{F S F}^{-1}=\mathcal{G}^{-}\left(\mathcal{G}^{+}\right)^{*},
$$

where

$$
\mathcal{G}^{ \pm}=\mathcal{F}\left(\Omega^{ \pm}\right)^{*}
$$

are the distorted Fourier transforms, so called because they intertwine $P$ with multiplication by $|\xi|^{2}:|\xi|^{2} \mathcal{G}^{ \pm}=\mathcal{G}^{ \pm} P$. It is not hard to show that $\mathcal{F} S \mathcal{F}^{-1}$ commutes with multiplication by functions of $|\xi|$. Therefore, it acts by

$$
\mathcal{F S F}^{-1} f(\lambda, \omega)=S(\lambda) f(\lambda, \omega), \quad \xi=\lambda \omega, \quad \omega \in S^{N-1}, \lambda>0,
$$

with $S(\lambda)$ a unitary operator on $L^{2}\left(S^{D-1}\right)$ for a.e. $\lambda$. We will call the family $S(\lambda)$ the analytic scattering matrix.

The geometric scattering matrix is defined via the large distance behaviour (as compared to large time behaviour) of solutions of the time independent equation $P \psi=\lambda^{2} \psi$. It is well known ([11], Lecture 2) that for every smooth function, $g$, on the sphere at infinity, there is a unique generalized eigenfunction of $P$ with energy $\lambda^{2}$ of the form

$$
|z|^{-\frac{D-1}{2}}\left(e^{-i \lambda|z|} g(\hat{z})+e^{+i \lambda|z|} h(\hat{z})\right)+O\left(|z|^{\frac{D+1}{2}}\right),|z| \rightarrow \infty, \hat{z}=z /|z|,
$$

with $h$ smooth and uniquely determined by $g$. The function $g$ is called the incoming data, and $h$ the outgoing data, of the generalized eigenfunction. The reason for the terms incoming and outgoing is that, if this function is multiplied by $e^{-i \lambda^{2} t}$ then we get a solution of the time dependent equation, and the exponentials multiplying the coefficients $g$ and $h$ are then $e^{-i \lambda(|z|+\lambda t)}$ and $e^{i \lambda(|z|-\lambda t)}$, which are spherical incoming and outgoing waves, respectively.

The geometric scattering matrix (defined in [11, where it is called the absolute scattering matrix) is the map

$$
\tilde{S}(\lambda): g \mapsto h .
$$

This extends to a unitary map on $L^{2}\left(S^{D-1}\right)$ for every $\lambda>0$. The Schwartz kernel $\tilde{S}(\lambda)(\omega, \theta)$ can be read off explicitly from the asymptotic expansion as $z=r \theta \rightarrow \infty$ of the plane waves (Lippman-Schwinger eigenfunctions) $u_{\omega, \lambda}(z)$, which are those 
eigenfunctions which are suitable perturbations of the free plane waves $e^{-i \lambda z \cdot \omega}$. They correspond in (3) to $g$ a delta function supported at $\omega \in S^{D-1}$.

These two families of operators are not identical; they differ by normalization. This can easily be seen by calculating the scattering matrices for the free Laplacian. Then it is obvious that the analytic scattering matrix is the identity. On the other hand, the geometric scattering matrix is equal to $i^{-D+1} R$, where $R$ is the antipodal map on $S^{D-1}$. Thus, the claim that these two scattering matrices 'coincide' should be understood to mean that they differ only by normalization: in other words, that

$$
\tilde{S}(\lambda)=i^{-D+1} S(\lambda) R
$$

Since $S$ satisfies the reciprocity relation, it does not matter on which side we multiply by $R$. As noted above, equation (4) is well known for the two-body operator (see e.g. [14, p. 107).

Now let us turn to generalized $N$ body Schrödinger operators. By definition, a generalized $N$ body Schrödinger operator is a differential operator $H$ on $\mathbb{R}^{D}$ which takes the form

$$
H=\Delta+V, \quad V=\sum_{k=1}^{M} \pi_{k}^{*}\left(V_{k}\right),
$$

where $\pi_{k}$ is a projection to a subspace $X^{k}$ of dimension $d_{k}, 1 \leq d_{k} \leq D-1$, and $V_{k}$ is a function on $X^{k}$. In this paper we assume that the 'two-body potentials' $V_{k}$ are of Schwartz class on $X^{k}$. If this assumption holds we will say that the two-body potentials are 'very short range'. The orthogonal complement of $X^{k}$ is denoted $X_{k}$ and called the $k$-th collision plane. We say that $H$ is a generalized three-body Schrödinger operator if the $X_{k}$ intersect pairwise only at the origin.

The main example, of course, is the reduced Schrödinger operator for $N$ particles moving in $\mathbb{R}^{d}$ under pairwise potential iterations. The Hamiltonian for the particles takes the form

$$
\sum_{j=1}^{N} \Delta_{z_{j}}+\sum_{1 \leq i<j \leq N} V_{i j}\left(z_{i}-z_{j}\right), \quad z_{i} \in \mathbb{R}^{d} .
$$

After reducing by the symmetry $z_{i} \rightarrow z_{i}+a, a \in \mathbb{R}^{d}$ (physically this symmetry is the source of momentum conservation), we get an operator of the form (5) in $\mathbb{R}^{(N-1) d}$ where the collision planes have codimension $d$. If $N=3$ then the collision planes are pairwise transverse.

The main difference between the two-body problem and the $N$ body problem is that, in the $N$ body problem, $N \geq 3$, the total potential $V$ does not decay to zero at infinity. Equation (5) shows that the potential $V_{k}$ is constant along affine planes parallel to $X_{k}$. Therefore, if we compactify $\mathbb{R}^{D}$ with a $D-1$ sphere at infinity, and use $1 /|z|$ as a boundary defining function, then $V$ does not decay at the intersection between the sphere at infinity and the collision planes $X_{k}$. We will denote this intersection $C_{k}$ and write $C=\bigcup_{k} C_{k}$, and call $C_{k}$ the $k$-th cluster set. With the very short range assumption, notice that $V$ is rapidly decreasing in any open cone in $\mathbb{R}^{D}$ disjoint from the collision planes.

For the $N$ body problem, it has been known for some time that the free channel wave operators (11) exist ([14], theorem XI.35), so the free channel analytic scattering matrix is well defined by (11) and (2). More recently, Vasy [17] showed that the geometric scattering matrix exists, by showing that there is a representation (3) 
for generalized eigenfunctions in a suitable sense: namely, that if $g$ is smooth and is supported away from the cluster sets, then there is a unique eigenfunction with the asymptotics (3) away from the cluster sets, with $h$ smooth on $S^{D-1} \backslash C$ and uniquely determined by $g$. Thus, the geometric scattering matrix is well-defined as an operator on $S^{D-1} \backslash C$. One would like to know that the $N$ body analytic and geometric scattering matrices bear the same relation (4) that they do in the two-body case. In the rest of this paper we prove that this is the case.

Remark. Since the first version of this note appeared, Vasy [19] has generalized the result, using a different method, to cover all channels for $N$ body Schrödinger operators.

\section{THE MAIN THEOREM}

Let $H$ be a generalized $N$ body Schrödinger operator with very short range potentials. In this section we prove

Theorem 1. As an operator on $C^{\infty}\left(S^{D-1} \backslash C\right)$, the free channel geometric scattering matrix $\tilde{S}(\lambda)$ is related to the free channel analytic scattering matrix $S(\lambda)$ by

$$
\tilde{S}(\lambda)=i^{-D+1} S(\lambda) R
$$

The proof of this result is in three steps. First, we show that the generalized eigenfunctions of Herbst-Skibsted are the same as the plane waves constructed in [4] when the potentials are very short range. Second, we show that the analytic scattering matrix satisfies the usual equation involving the transition matrix (18). Finally, we show that the geometric scattering matrix also satisfies this equation.

Step 1. Let $R(\mu)$ denote the resolvent $(H-\mu)^{-1}, \operatorname{Im} \mu \neq 0$, and let $R\left(\lambda^{2}+i 0\right)$ denote the limit $R\left(\lambda^{2}+i \epsilon\right)$ as $\epsilon \rightarrow 0$ for $\lambda \in \mathbb{R}$, which by [13] exists as an operator from $\langle z\rangle^{-a} L^{2}$ to $\langle z\rangle^{a} L^{2}$ for $a>1 / 2$. Denote by $G\left(y, z ; \lambda^{2}\right)$ the Schwartz kernel of $R\left(\lambda^{2}+i 0\right)$. The generalized eigenfunctions of Herbst-Skibsted [6] are defined by

$$
f(\xi ; z)=\lim _{r \rightarrow \infty} r^{(D-1) / 2} e^{-i \lambda r} G\left(r \omega, z ; \lambda^{2}\right), \quad \xi=\lambda \omega .
$$

Herbst and Skibsted show uniform convergence on compact subsets of $\mathbb{R}_{\xi}^{D} \times \mathbb{R}_{z}^{D}$, provided $\xi$ is kept away from the collision planes.

We show that the limit is equal to a constant times the plane wave $u_{\omega, \lambda}$ constructed in [4] (for $\omega \notin C)$. Let $\psi \in C_{c}^{\infty}\left(\mathbb{R}^{D}\right)$ and let $\chi=R\left(\lambda^{2}+i 0\right) \psi$. Then

$$
\chi(y)=\int G\left(y, z ; \lambda^{2}\right) \psi(z) d z .
$$

By the commutator argument in [10] or [17], for $\hat{z}=z /|z|$ away from $C, \chi$ has the behaviour

$$
\begin{gathered}
\chi(r \hat{z})=r^{-(D-1) / 2} e^{i \lambda r} a(\hat{z})+o\left(r^{-(D-1) / 2}\right), \\
\left(\partial_{r}-i \lambda\right) \chi(z)=o\left(r^{-(D-1) / 2}\right) .
\end{gathered}
$$

Therefore, for $\omega \notin C$, we have

$$
a(\omega)=\lim _{r \rightarrow \infty} r^{(D-1) / 2} e^{-i \lambda r} \int G(r \omega, z ; \lambda) \psi(z) d z=\int f(r \omega ; z) \psi(z) d y .
$$

Consider

$$
\int u_{\omega, \lambda}(z) \psi(z) d z
$$


We will show that this is equal to a fixed constant times $a(\omega)$, proving the result. Since the details are somewhat intricate, let us first give a proof of this result which is valid for rapidly decreasing two-body potentials (but not $N$ body potentials for $N \geq 3$ ), and then give the general proof.

In the two-body case, the plane wave is $u_{\omega, \lambda}=e^{-i \lambda z \cdot \omega}-R\left(\lambda^{2}+i 0\right)\left(V e^{-i \lambda z \cdot \omega}\right)$. Therefore,

$$
\begin{gathered}
\left\langle u_{\omega, \lambda}, \bar{\psi}\right\rangle=\left\langle e^{-i \lambda z \cdot \omega}, \bar{\psi}\right\rangle-\left\langle R\left(\lambda^{2}+i 0\right)\left(V e^{-i \lambda z \cdot \omega}\right), \bar{\psi}\right\rangle \\
=\left\langle e^{-i \lambda z \cdot \omega}, \bar{\psi}\right\rangle-\left\langle e^{-i \lambda z \cdot \omega}, V R\left(\lambda^{2}-i 0\right) \bar{\psi}\right\rangle \\
=\left\langle e^{-i \lambda z \cdot \omega},\left(\Delta-\lambda^{2}\right) \bar{\chi}\right\rangle
\end{gathered}
$$

since $\left(\Delta-\lambda^{2}\right) \chi=\psi+V R\left(\lambda^{2}+i 0\right) \psi$. Since $\left(\Delta-\lambda^{2}\right) \chi \in \mathcal{S}$, in the two-body case, we can use Green's theorem to get

$$
\begin{aligned}
& \left\langle e^{-i \lambda z \cdot \omega},\left(\Delta-\lambda^{2}\right) \bar{\chi}\right\rangle=\lim _{r \rightarrow \infty} \int_{|z| \leq r} e^{-i \lambda z \cdot \omega}\left(\Delta-\lambda^{2}\right) \chi d z \\
& =\lim _{r \rightarrow \infty} \int_{|z|=r}\left(\frac{\partial e^{-i \lambda z \cdot \omega}}{\partial \nu} \chi(z)-e^{-i \lambda z \cdot \omega} \frac{\partial \chi(z)}{\partial \nu}\right) d \sigma(z) .
\end{aligned}
$$

Given (8), we can use stationary phase to directly compute the limit. From the stationary point at $\hat{z}=\omega$ we get a contribution

$$
2 i \lambda\left(\frac{2 \pi i}{\lambda}\right)^{(D-1) / 2} a(\omega)
$$

and from the stationary point at $\hat{z}=-\omega$ we get zero because the contributions from the two terms cancel. However, in preparation for the $N$ body case, where we have far less explicit information about the function $\chi$ near the cluster set, it is useful to derive this vanishing result in another way. Choose a cutoff function $\phi(\hat{z})$, where $\phi$ is equal to one in a neighbourhood of $\omega$ and zero in a neighbourhood of $C$. If we insert $\phi$ and then $(1-\phi)$ into the integral (11), then the $\phi$ term yields the value (12), and we will show via the scattering wavefront set (see appendix $\mathrm{A}$ ) that the limit of the integral with the factor $1-\phi$ is zero. Notice that $(1-\phi) e^{-i \lambda z \cdot \omega}$ has scattering wavefront set contained in $\{\tau<\lambda-\delta\}$ for some $\delta>0$, by construction. From (88) it is easy to see that $\chi$ has scattering wavefront set contained in $\{\tau=-\lambda\}$. Since taking normal derivatives does not increase the scattering wavefront set, by (53) the integrand of (11) with the factor $(1-\phi)$ included has scattering wavefront set contained in $\{\tau<0\}$. Also, we know a priori that the limit as $r \rightarrow \infty$ of the integral exists. The fact that the limit is zero then follows from the following lemma.

Lemma 2 (The zero limit lemma). If $\lim _{r \rightarrow \infty} \int_{|z|=r} f$ exists and if ${ }^{s c} W F(f) \subset$ $\{\tau \neq 0$ or $\mu \neq 0\}$, then the limit is zero.

Proof. This is a simple application of the pushforward theorem for the scattering wavefront set (Theorem 9, section 4). The integral over spheres is equivalent to the pushforward from $\mathbb{R}^{N}$ to $\mathbb{R}$ with $\pi$ mapping $z$ to $|z|$, using the Riemannian density to define the pushforward. Let $g(r)$ be a smooth function on $\mathbb{R}$ which is equal to the value of this integral for large $r$. If $\lim _{r \rightarrow \infty} g(r)$ exists and is nonzero, then the Fourier transform of $g$ is singular at zero. This implies, using (52), that $(+1, \tau=0)$ is contained in the scattering wavefront set of $g$ (here +1 represents the point at the boundary of $B^{1}$ representing $r=\infty$ ). But this contradicts Theorem 9 (see (47) and (48)). Therefore the limit must be zero. 
In the $N$ body case, we use the plane waves constructed in 4 . There it was shown that for $\omega \in S^{D-1}$ not in the cluster set, one can make sense of $R\left(\lambda^{2}+i 0\right)\left(V e^{-i \lambda z \cdot \omega}\right)$. The plane waves were then defined by

$$
u_{\omega, \lambda}=e^{-i \lambda z \cdot \omega}-R\left(\lambda^{2}+i 0\right)\left(V e^{-i \lambda z \cdot \omega}\right),
$$

and were shown to satisfy the estimate

$$
{ }^{s c} \mathrm{WF}\left(u_{\omega, \lambda}-e^{-i \lambda z \cdot \omega}\right) \subset\{\tau<\lambda-\delta\}
$$

for some $\delta>0$ depending on the distance between $\omega$ and $C$.

In the previous argument, we can no longer assert that

$$
\left\langle R\left(\lambda^{2}+i 0\right)\left(V e^{-i \lambda z \cdot \omega}\right), \bar{\psi}\right\rangle=\left\langle e^{-i \lambda z \cdot \omega}, V R\left(\lambda^{2}-i 0\right) \bar{\psi}\right\rangle
$$

since the right hand side is not well defined. To overcome this difficulty we use a cutoff function. Thus, we show that $R\left(\lambda^{2}+i 0\right)\left(V e^{-i \lambda z \cdot \omega}\right)$ is the distributional limit of

$$
R\left(\lambda^{2}+i 0\right)\left(\rho\left(\frac{r}{R}\right) V e^{-i \lambda z \cdot \omega}\right), \quad r=|z|,
$$

as $R \rightarrow \infty$, where $\rho(t) \in C_{c}^{\infty}(\mathbb{R})$ is identically equal to one when $|t| \leq 1$ and supported in $|t|<2$. To see this, we need to consider the operators $F_{+}(B)$ and $\tilde{F}_{-}(B)=\operatorname{Id}-F_{+}(B)$ where $B$ is the operator introduced by Gérard, Isozaki and Skibsted [1], [2] (see (54) ) and $F_{+}$is a function that is zero on $(-\infty,-\lambda)$ and one on $(-\lambda+\delta, \infty)$. These were and also play an important role in [4]. For more details see appendix B. If we write

$$
R\left(\lambda^{2}+i 0\right)\left(\rho\left(\frac{r}{R}\right) V e^{-i \lambda z \cdot \omega}\right)=R\left(\lambda^{2}+i 0\right)\left(F_{+}(B)+\tilde{F}_{-}(B)\right)\left(\rho\left(\frac{r}{R}\right) V e^{-i \lambda z \cdot \omega}\right),
$$

then the term with $F_{+}(B)$ converges in $\mathcal{S}^{\prime}$ by the adjoint of equation (2) of Theorem 2.10 of $\left[2\right.$. If we commute the $\tilde{F}_{-}(B)$ and $\rho(r / R)$ terms, then we get convergence because $\tilde{F}_{-}(B)\left(V e^{-i \lambda z \cdot \omega}\right)$ is in $\langle z\rangle^{-\infty} L^{2}$ (see [4], section 5), and the commutator itself can be handled using the commutator expansion as in Lemma 10.

Therefore,

$$
\begin{aligned}
\int u_{\omega, \lambda}(z) \psi(z) d z & =\lim _{R \rightarrow \infty} \int\left(e^{-i \lambda z \cdot \omega}-R\left(\lambda^{2}+i 0\right)\left(\rho\left(\frac{r}{R}\right) V e^{-i \lambda z \cdot \omega}\right)\right) \psi(z) d z \\
& =\lim _{R \rightarrow \infty} \int e^{-i \lambda z \cdot \omega} \rho\left(\frac{r}{R}\right) \psi(z) d z-\int\left(\rho\left(\frac{r}{R}\right) V e^{-i \lambda z \cdot \omega}\right) \chi(z) d z \\
& =\lim _{R \rightarrow \infty} \int e^{-i \lambda z \cdot \omega} \rho\left(\frac{r}{R}\right)\left(\Delta-\lambda^{2}\right) \chi(z) d z
\end{aligned}
$$

since $\psi-V \chi=\left(\Delta-\lambda^{2}\right) \chi$. Integrating by parts, this is equal to

$$
\int R^{-1} \rho^{\prime}\left(\frac{r}{R}\right)\left(\frac{\partial e^{-i \lambda z \cdot \omega}}{\partial \nu} \chi(z)-e^{-i \lambda z \cdot \omega} \frac{\partial \chi(z)}{\partial \nu}\right) d z+\int(\Delta \rho(|z| / R)) e^{-i \lambda z \cdot \omega} \chi(z) d z
$$

As in the previous section, we insert $\phi(\hat{z})+(1-\phi(\hat{z}))$. With the factor $\phi$, it is easy to show via stationary phase that we get (12). With the factor $1-\phi$, we will show via the zero limit lemma that the limit is zero. Notice that we know a priori that the integral (15) with the factor $(1-\phi)$ has a limit as $R \rightarrow \infty$. 
To apply the zero limit lemma we need to express (15) as an integral over the sphere of radius $R$. Define

$$
f_{1}(z)=(1-\phi(\hat{z}))\left(\frac{\partial e^{-i \lambda z \cdot \omega}}{\partial \nu} \chi(z)-e^{-i \lambda z \cdot \omega} \frac{\partial \chi(z)}{\partial \nu}\right)
$$

and

$$
f_{2}(z)=(1-\phi(\hat{z})) e^{-i \lambda z \cdot \omega} \chi(z) .
$$

Notice that $(1-\phi) e^{-i \lambda z \cdot \omega}$ has scattering wavefront set contained in $\{\tau<\lambda-\delta\}$ for some $\delta>0$, by construction. As for $\chi$, by Theorem 2.10 of [1] we have $F_{-}(B) \chi \in$ $\langle z\rangle^{-\infty} L^{2}$ and using the method of [4, section 5, one can improve this estimate to show ${ }^{s c} \mathrm{WF}(\chi) \subset\{\tau \leq-\lambda\}$. It follows that $f_{1}$ and $f_{2}$ have scattering wavefront set contained in $\{\tau<0\}$.

The integral (15), with factor $(1-\phi)$ inserted, may be written as a boundary integral

$$
\int_{|z|=R}\left(\tilde{f}_{1}+\tilde{f}_{2}\right) d \sigma(z),
$$

where

$$
\tilde{f}_{1}(z)=\int_{1}^{2} \rho^{\prime}(t) t^{D-1} f(t z) d t
$$

and $\tilde{f}_{2}$ has a similar expression. The functions $\tilde{f}_{1}$ and $\tilde{f}_{2}$ may be expressed as pushforwards of $\rho^{\prime}(t) t^{D-1} f(t z)$ under the pushforward map

$$
\left[\overline{\mathbb{R}^{D}} \times[0,2]_{s} ; S^{D-1} \times\{s=0\}\right] \rightarrow \overline{\mathbb{R}^{D}}, \quad s=R^{-1}, t=r / R,
$$

and the pushforward theorem then shows ${ }^{s c} \mathrm{WF}\left(\tilde{f}_{i}\right) \subset\{\tau<0\}$. (The blowup of the corner in the space above ensures that the function is nontrivial at only one boundary face so that the pushforward theorem applies.) Thus, by the zero limit lemma, the integral (15) with factor $(1-\phi)$ is zero.

Therefore, the integral (10) is equal to $2 i \lambda a(\omega)$. By (9) then,

$$
\int u_{\omega, \lambda}(z) \psi(z) d z=2 i \lambda\left(\frac{2 \pi i}{\lambda}\right)^{(D-1) / 2} \int f(\lambda \omega ; z) \psi(z) d z
$$

for all $\psi \in C_{c}^{\infty}$. We conclude

$$
u_{\omega, \lambda}=2 i^{(D+1) / 2} \lambda^{-(D-3) / 2}(2 \pi)^{(D-1) / 2} f(\lambda \omega, \cdot)=(2 \pi)^{D / 2} \overline{e(\lambda \omega, \cdot)}
$$

where $\overline{e(\lambda \omega, \cdot)}$ are the normalized eigenfunctions of Herbst and Skibsted. Herbst and Skibsted showed that they form the Schwartz kernel of the free channel distorted Fourier transform. (Jensen and Kitada 9 had previously constructed the Schwartz kernel of the distorted Fourier transform for $N$ body Schrödinger operators, but only obtained generalized eigenfunctions for a.e. $\xi=\lambda \omega$.) This completes Step 1 of the proof.

Step 2. In this step we show that the analytic scattering matrix satisfies Theorem XI.42 in [14, p. 107 where $\phi(x, k)$ are the plane waves. We need to use step 1 , and 6], which show that the distorted Fourier transforms have kernels given by the plane waves $u_{\omega, \lambda}$.

Define the transition matrix by

$$
T\left(k, k^{\prime}\right)=(2 \pi)^{-D / 2} \mathcal{F}_{k \leftarrow z}\left(V(z) u_{-k^{\prime}}(z)\right) .
$$


NB: we use here the convention

$$
(\mathcal{F} f)(k)=(2 \pi)^{-D / 2} \int_{\mathbb{R}^{D}} e^{-i z \cdot k} f(z) d z
$$

for the Fourier transform, so that it is a unitary operator. We adopt the notation of Reed and Simon; $k=\omega \lambda$ from the previous section. (However, we use different conventions for the sign of the Lippman-Schwinger eigenfunctions, and for the sesquilinear form, from Reed and Simon.) We also write $\mathcal{F}_{k \leftarrow z}$ to indicate which variables are being used. The next result asserts that Reed and Simon's Theorem XI.42 holds for the $N$ body problem with very short range potentials.

Proposition 3. Let $f$ and $g$ be Schwartz functions whose Fourier transforms have support disjoint from the collision planes. Then the free channel analytic scattering matrix $S$ satisfies

$$
\langle(S-\mathrm{Id}) f, g\rangle=-\frac{i \pi}{|k|} \int_{|k|=\left|k^{\prime}\right|} T\left(k, k^{\prime}\right) \hat{f}\left(k^{\prime}\right) \overline{\hat{g}(k)} d \sigma(k) d k^{\prime} .
$$

Proof. The proof follows that given in Reed and Simon almost exactly. We just need to justify carefully the last step.

Since asymptotic completeness holds for the $N$ body Hamiltonian under our conditions [15], the free channel wave operators $\Omega^{ \pm}$defined by (11) exist. In [6] it is shown that $\mathcal{G}^{ \pm}=\mathcal{F}\left(\Omega^{ \pm}\right)^{*}$ is a unitary operator from ran $\Omega^{ \pm}$to $L^{2}\left(\mathbb{R}^{D}\right)$, with $\mathcal{G}^{-}$having Schwartz kernel $(2 \pi)^{-D / 2} u_{k}(z)$ and $\mathcal{G}^{+}$having Schwartz kernel $(2 \pi)^{-D / 2} \overline{u_{-k}(z)}$. Thus, we have

$$
\begin{aligned}
\langle(S-\mathrm{Id}) f, g\rangle & =\left\langle\left(\Omega^{-}-\Omega^{+}\right)^{*} \Omega^{+} f, g\right\rangle \\
& =\left\langle\Omega^{+} f,\left(\Omega^{-}-\Omega^{+}\right) g\right\rangle \\
& =\lim _{T \rightarrow \infty} \int_{-T}^{T}\left\langle\Omega^{+} f, e^{i H t}(i V) e^{-i \Delta t} g\right\rangle d t \\
& =\lim _{\epsilon \rightarrow 0} \int_{-\infty}^{\infty} e^{-\epsilon|t|}\left\langle\Omega^{+} f, e^{i H t}(i V) e^{-i \Delta t} g\right\rangle d t \\
& =\lim _{\epsilon \rightarrow 0} i \int_{-\infty}^{\infty} e^{-\epsilon|t|}\left\langle\mathcal{G}^{+} \Omega^{+} f, \mathcal{G}^{+} e^{i H t}(i V) e^{-i \Delta t} g\right\rangle d t
\end{aligned}
$$

The introduction of the Abelian limit in the fourth line is justified by Lemma 5 , p. 95, of [14] and the last line is justified by the fact that $\left(\mathcal{G}^{+}\right)^{*} \mathcal{G}^{+} \Omega^{+}=\Omega^{+}$. We have $\mathcal{G}^{+} \Omega^{+} f=\hat{f}$. The right member of the inner product we rewrite using the formulae

$$
\mathcal{G}^{+}=\mathcal{F}\left(\Omega^{+}\right)^{*} \text { and }\left(\Omega^{+}\right)^{*} e^{i t H}=e^{i t \Delta}\left(\Omega^{+}\right)^{*}
$$

to get

$$
\left(\mathcal{F} e^{i t \Delta}\left(\Omega^{+}\right)^{*}(i V) e^{-i t \Delta} g\right)\left(k^{\prime}\right)=e^{i t\left|k^{\prime}\right|^{2}}\left(\mathcal{G}^{+}\right)_{k^{\prime} \leftarrow z}(i V)(z) \mathcal{F}_{z \leftarrow k}^{-1} e^{-i t|k|^{2}} \hat{g}(k) .
$$

Therefore (19) is equal to

$$
\left\langle\mathcal{F}_{k \leftarrow z}(-i V(z)) \mathcal{G}_{z \leftarrow k^{\prime}}^{+} e^{-i t\left|k^{\prime}\right|^{2}} \hat{f}\left(k^{\prime}\right), e^{-i t|k|^{2}} \hat{g}(k)\right\rangle .
$$

Since the kernel of $\left(\mathcal{G}^{+}\right)_{z \leftarrow k^{\prime}}^{*}$ is $(2 \pi)^{-D / 2} u_{-k^{\prime}}(z)$, this is the same as

$$
-i\left\langle\int d k^{\prime} T\left(k, k^{\prime}\right) e^{-i t\left|k^{\prime}\right|^{2}} \hat{f}\left(k^{\prime}\right), e^{-i t|k|^{2}} \hat{g}(k)\right\rangle .
$$


Therefore, (19) is equal to

$$
-i \lim _{\epsilon \rightarrow 0} \int_{-\infty}^{\infty} d t e^{-\epsilon|t|} \int d k^{\prime} \int d k e^{i t\left(|k|^{2}-\left|k^{\prime}\right|^{2}\right)} T\left(k, k^{\prime}\right) \hat{f}\left(k^{\prime}\right) \overline{\hat{g}(k)} .
$$

Performing the $t$ integral, we get

$$
-i \lim _{\epsilon \rightarrow 0} \int d k^{\prime} \int d k \frac{2 \epsilon}{\left(|k|^{2}-\left|k^{\prime}\right|^{2}\right)^{2}+\epsilon^{2}} T\left(k, k^{\prime}\right) \hat{f}\left(k^{\prime}\right) \overline{\hat{g}(k)} .
$$

Since, in the sense of distributions,

$$
\frac{2 \epsilon}{\left(|k|^{2}-\left|k^{\prime}\right|^{2}\right)^{2}+\epsilon^{2}} \rightarrow \frac{\pi}{|k|} \delta\left(|k|-\left|k^{\prime}\right|\right) \text { as } \epsilon \rightarrow 0,
$$

we would like to deduce that this converges to the desired quantity, (18). This requires justification because $T\left(k, k^{\prime}\right)$ is itself a distribution. Herbst-Skibsted [6] showed that $u_{k^{\prime}}(z)$ is smooth in $k^{\prime}$ for $k^{\prime}$ not in a collision plane, so we may regard $\hat{f} \cdot T$ as a distribution in $k$ parametrized smoothly by $k^{\prime}$. Let us fix $k^{\prime}$. We observe that the the limit (24) exists in $\mathcal{D}^{\prime}(\Gamma)$, the space of distributions with wavefront set contained in $\Gamma$, the conormal bundle of the submanifold $\left\{|k|=\left|k^{\prime}\right|\right\}$. Thus, by [7], theorem 8.2.13, the limit gives (18) provided that $\mathrm{WF}\left(\hat{f}\left(k^{\prime}\right) T\left(\cdot, k^{\prime}\right) \overline{\hat{g}(\cdot)}\right)$ is disjoint from $\Gamma$. Therefore, the next lemma completes the proof.

Lemma 4. Let $k^{\prime}$ be a fixed vector which is not in any collision plane, and assume that the support of $\hat{g}$ is disjoint from the collision planes. Then the wavefront set of $T\left(k, k^{\prime}\right) \overline{\hat{g}(k)}$ does not intersect the conormal bundle of $\left\{|k|=\left|k^{\prime}\right|\right\}$.

Proof. Since $V_{i}$ are rapidly decreasing two-body potentials, the scattering wavefront set of $V u_{-k^{\prime}}$ is contained in the set

$$
\{(\hat{z}, \zeta) \mid \hat{z} \in C\} \text {. }
$$

Therefore, the Fourier transform has wavefront set contained in (see [52) )

$$
\{(k,-t \hat{z}) \mid \hat{z} \in C, t>0\} .
$$

Thus, if the support of $g$ is disjoint from collision planes, then

$$
\mathrm{WF}\left(T\left(k, k^{\prime}\right) \overline{\hat{g}(k)}\right) \subset\{(k,-t \hat{z}) \mid k \notin C, \hat{z} \in C\} .
$$

This is disjoint from the conormal bundle of $|k|=\left|k^{\prime}\right|$, as required.

Remark. This lemma shows that the restriction of $T \overline{\hat{g}}$ to $\left\{|k|=\left|k^{\prime}\right|\right\}$ in (18) is well defined.

Step 3. Proposition 3 shows that the Schwartz kernel of $S(\lambda)$ - Id, regarded as an operator on $S^{D-1} \backslash C$, is

$$
-\frac{i \pi}{\lambda} T(\lambda \theta, \lambda \omega), \quad \theta, \omega \in S^{D-1} \backslash C .
$$

In this step we complete the proof by showing that the geometric scattering matrix satisfies

$$
\tilde{S}(\lambda)-i^{-D+1} R=\frac{i^{-D} \pi}{\lambda} T(\lambda) R
$$

away from collision planes, showing that the analytic and geometric scattering matrices satisfy the relation (44). 
To compute $\tilde{S}(\lambda)(g)$, we form the generalized eigenfunction with energy $\lambda^{2}$ and incoming data $g(\hat{z})$, supported away from the cluster set. This is equal to

$$
u_{g, \lambda}=\left(\frac{\lambda}{2 \pi}\right)^{\frac{D-1}{2}} e^{-i \pi \frac{D-1}{4}} \int u_{\omega, \lambda}(z) g(\omega) d \omega .
$$

To see this, write $u_{\omega, \lambda}=\phi(\hat{z}) e^{-i \lambda z \cdot \omega}+(1-\phi(\hat{z})) e^{-i \lambda z \cdot \omega}+u_{\omega, \lambda}^{\prime}$ with $\phi$ equal to one near $\hat{z}=\omega$ and zero near $-\omega$. Then, by stationary phase, we have

$$
\lim _{r \rightarrow \infty} r^{-\frac{D-1}{2}} e^{i \lambda r}\left(\frac{\lambda}{2 \pi}\right)^{\frac{D-1}{2}} e^{-i \pi \frac{D-1}{4}} \int_{|z|=r} \phi(\hat{z}) e^{-i \lambda z \cdot \omega} g(\omega) d \omega=g(\hat{z}),
$$

and

$$
\lim _{r \rightarrow \infty} r^{-\frac{D-1}{2}} e^{-i \lambda r}\left(\frac{\lambda}{2 \pi}\right)^{\frac{D-1}{2}} e^{-i \pi \frac{D-1}{4}} \int_{|z|=r}(1-\phi(\hat{z})) e^{-i \lambda z \cdot \omega} g(\omega) d \omega=i^{-D+1} g(-\hat{z})
$$

which gives us the 'free' term $i^{-D+1} R g$. We must show that the limit

$$
\lim _{r \rightarrow \infty} r^{-\frac{D-1}{2}} e^{-i \lambda r}\left(\frac{\lambda}{2 \pi}\right)^{\frac{D-1}{2}} e^{-i \pi \frac{D-1}{4}} \int_{|z|=r} u_{\omega, \lambda}^{\prime}(z) g(\omega) d \omega
$$

exists, which therefore gives us $\left(\tilde{S}(\lambda)-i^{-D+1} R\right) g$, and show that it satisfies (25).

We do this by analyzing the distributional limit of $r^{-\frac{D-1}{2}} e^{-i \lambda r} u_{\omega, \lambda}^{\prime}(r \hat{z})$. Given a test function $\psi \in C_{c}^{\infty}\left(S^{D-1} \backslash C\right)$, consider

$$
\lim _{r \rightarrow \infty} r^{-(D-1) / 2} e^{-i \lambda r} \int_{|z|=r} u_{\omega, \lambda}^{\prime}(z) \psi(\hat{z}) d \sigma(z) .
$$

Define

$$
\Psi(z)=(2 \pi)^{1 / 2} \lambda^{-\frac{D-1}{2}} e^{-i \pi \frac{D-1}{4}} \mathcal{F}_{z \leftarrow k}^{-1}(\psi(-\hat{k}) \delta(|k|-\lambda)), \quad \hat{k}=k /|k|,
$$

so $\left(\Delta-\lambda^{2}\right) \Psi(z)=0$. Then $\Psi$ has an asymptotic expansion, by stationary phase

$$
\begin{gathered}
\Psi(z) \sim e^{-i \lambda r}\left(\sum_{k=0}^{\infty} r^{-\frac{D-1}{2}-k} a_{-, k}(\hat{z})\right)+e^{+i \lambda r}\left(\sum_{k=0}^{\infty} r^{-\frac{D-1}{2}-k} \alpha_{+, k}(\hat{z})\right) \\
a_{ \pm, k} \in C^{\infty}\left(S^{D-1}\right), \quad a_{-, 0}=\psi
\end{gathered}
$$

and the $a_{ \pm, j}$ given by universal differential operators applied to $a_{ \pm, 0}$, so in particular have support at infinity disjoint from the cluster sets. We need to decompose $\Psi$ into incoming and outgoing parts. To this end, choose a smooth function $\chi(t)$ with $\chi(\lambda)=1$ and supported in $[\lambda / 2,2 \lambda]$, and define

$$
\Psi_{ \pm}(z)= \pm(8 \pi)^{-\frac{1}{2}} \lambda^{-\frac{D+1}{2}} e^{i \pi \frac{D-3}{4}} \mathcal{F}_{z \leftarrow k}^{-1}\left(\psi(-k) \chi(|k|)\left(|k|^{2}-\lambda^{2} \mp i 0\right)^{-1}\right),
$$

so $\Psi=\Psi_{+}+\Psi_{-}$. Note that $\left(\Delta-\lambda^{2}\right) \Psi_{ \pm} \in \mathcal{S}$, and $\Psi_{ \pm}$have asymptotic expansions

$$
\Psi_{ \pm} \sim e^{ \pm i \lambda r} \sum_{k=0}^{\infty} r^{-\frac{N-1}{2}-j} a_{ \pm, k}(\hat{z})
$$

with the $a_{-, 0}(\hat{z})$ as above. 
Let us define

$$
\begin{aligned}
b(\psi, r)= & \int_{|z|=r} u_{\omega, \lambda}^{\prime}(z) \Psi(z) d \sigma(z)=b_{+}(\psi, r)+b_{-}(\psi, r), \\
& b_{ \pm}(\psi, r)=\int_{|z|=r} u_{\omega, \lambda}^{\prime}(z) \Psi_{ \pm}(z) d \sigma(z) .
\end{aligned}
$$

By the results of [4], the plane wave $u_{\omega, \lambda}$ is an element of the weighted Sobolev space $\langle z\rangle^{D / 2+1+\epsilon} H^{k}$, for any $k$. By the Sobolev embedding theorem, then, $u_{\omega, \lambda} \in$ $\langle z\rangle^{D / 2+1+\epsilon} L^{\infty}$. Therefore, in considering the limit (29), we only need to consider a finite number of terms in the expansion of $\Psi$, and we may express (29) as

$$
b_{-}(\psi, r)-\sum_{j=1}^{2 D} r^{-j} b_{-}\left(\psi_{j}, r\right)+O\left(r^{-1}\right)
$$

for a finite collection of $C^{\infty}$ functions $\psi_{j}$ having support no larger than that of $\psi$. If we can show, for arbitrary $\psi$ supported away from the collision planes, that the limit of $b_{ \pm}(\psi, r)$ exists, then it follows from (33) that the limit of (29) exists and is equal to the limit of (33) for $b_{-}$. So we will concentrate on analyzing the limit of $b_{ \pm}(\psi, r)$ as $r \rightarrow \infty$.

The strategy is to turn the boundary integral into an integral over $\mathbb{R}^{D}$ via Green's theorem. Using the identity

$$
\frac{d b_{ \pm}}{d r}(\psi, r)=\int_{|z|=r}\left(\frac{\partial u_{\omega, \lambda}^{\prime}}{\partial \nu} \Psi_{ \pm}+u_{\omega, \lambda}^{\prime} \frac{\partial \Psi_{ \pm}}{\partial \nu}\right) d \sigma(z)+\frac{D-1}{r} b_{ \pm}(\psi, r)
$$

we obtain

$$
\frac{d b_{ \pm}}{d r} \mp 2 i \lambda b_{ \pm}=\int_{|z|=r}\left(\frac{\partial u_{\omega, \lambda}^{\prime}}{\partial \nu} \Psi_{ \pm}-u_{\omega, \lambda}^{\prime} \frac{\partial \Psi_{ \pm}}{\partial \nu}+2 u_{\omega, \lambda}^{\prime}\left(\frac{\partial}{\partial \nu} \mp i \lambda+\frac{D-1}{2 r}\right) \Psi_{ \pm}\right) d \sigma(z) .
$$

Notice that the first two terms are of a form which allows the application of Green's theorem. First however we need to absorb the other terms. Since we can expand $\left(\partial_{r} \mp i \lambda+(D-1) / 2 r\right) \Psi_{ \pm}$in the form

$$
e^{ \pm i \lambda r} \sum_{j \geq 2} r^{-(D-1) / 2-j} \tilde{a}_{ \pm, j}(\hat{z})
$$

we can write

$$
\frac{d b_{ \pm}}{d r} \mp 2 i \lambda b_{ \pm}=\int_{|z|=r}\left(\frac{\partial u_{\omega, \lambda}^{\prime}}{\partial \nu} \Psi_{ \pm}-u_{\omega, \lambda}^{\prime} \frac{\partial \Psi_{ \pm}}{\partial \nu}\right) d \sigma(z)+\sum_{j=2}^{2 D} r^{-j} b_{ \pm}\left(\tilde{\psi}_{j}, r\right)+O\left(r^{-1}\right) .
$$

Via Green's theorem, it is easy to see (and show below) that the integral on the right hand side has a limit as $r \rightarrow \infty$. Using this observation we can iteratively absorb the error terms $\sum r^{-j} b_{ \pm}\left(\tilde{\psi}_{j}, r\right)$. To do this, we construct

$$
c_{ \pm}(\psi, r)=b_{ \pm}(\psi, r)+\sum_{j=1}^{2 D} r^{-j} b_{ \pm}\left(k_{j}, r\right)
$$

such that

$$
\left(\frac{d}{d r} \mp 2 i \lambda\right) c_{ \pm}(\psi, r)=\left(\frac{d}{d r} \mp 2 i \lambda\right) b_{ \pm}(\psi, r)-\sum_{j=2}^{2 D} r^{-j} b_{ \pm}\left(\tilde{\psi}_{j}, r\right)+O\left(r^{-1}\right) .
$$


This is possible for suitable, unique $k_{j}$ which can be determined iteratively for $j=1,2, \ldots$ (for example, $k_{1}=\tilde{\psi}_{2}$ ). If we can show that $c_{ \pm}$has a limit as $r \rightarrow \infty$, then $b_{ \pm}$has the same limit, since it follows from (35) that we can express $b_{ \pm}(\psi, r)$ as a finite sum $c_{ \pm}(\psi, r)+\sum_{j \geq 1} r^{-j} c_{ \pm}\left(\tilde{k}_{j}, r\right)+O\left(r^{-1}\right)$. Thus it suffices to analyze $c_{ \pm}(\psi, r)$, which satisfies

$$
\frac{d c_{ \pm}}{d r} \mp 2 i \lambda c_{ \pm}=\int_{|z|=r}\left(\frac{\partial u_{\omega, \lambda}^{\prime}}{\partial \nu} \Psi_{ \pm}-u_{\omega, \lambda}^{\prime} \frac{\partial \Psi_{ \pm}}{\partial \nu}\right) d \sigma(z)+O\left(r^{-1}\right) .
$$

Applying Green's formula to (36) we find that

$$
\frac{d c_{ \pm}}{d r} \mp 2 i \lambda c_{ \pm}=\int_{|z| \leq r}\left(V(z) u_{\omega, \lambda}(z) \Psi_{ \pm}+u_{\omega, \lambda}^{\prime}\left(\Delta-\lambda^{2}\right) \Psi_{ \pm}\right) d z+O\left(r^{-1}\right) .
$$

Since $\Psi_{ \pm}$is rapidly decreasing in a conic neighbourhood of the collision planes and $\left(\Delta-\lambda^{2}\right) \Psi_{ \pm}$is rapidly decreasing everywhere, the right hand side has a limit as $r \rightarrow \infty$. But for $c_{+}$, we have in addition, by (35), that $c_{+}$is the pushforward of a sum of functions $r^{-j} u_{\omega, \lambda}^{\prime} \Psi_{j,+}$ each of which has the scattering wavefront bound

$$
{ }^{s c} \mathrm{WF}\left(r^{-j} u_{\omega, \lambda}^{\prime} \Psi_{j,+}\right) \subset\{\tau<-\delta\}
$$

by (13), (31), and (153). Therefore, by the zero limit lemma, the limit for $c_{+}$must be zero. Thus we may add the right hand side of (37) for the + sign to the right hand side for the - sign, obtaining

$$
c_{-}^{\prime}(r)+2 i \lambda c_{-}(r)=e(r) \text { with } \lim _{r \rightarrow \infty} e(r)=e(\infty) \equiv \int V(z) u_{\omega, \lambda}(z) \Psi(z) d z .
$$

We want to show that $2 i \lambda c_{-}$itself, and not just $c_{-}^{\prime}+2 i \lambda c_{-}$has the limit $e(\infty)$. This cannot be deduced from (38) alone because the homogeneous solution to the ODE on the left hand side is $e^{-2 i r}$ which has no limit as $r \rightarrow \infty$. However, we have one extra, crucial piece of information: by (13), (31) and (53), the functions $b_{-}$and therefore $c_{-}$have scattering wavefront set contained in $\{\tau \leq 2 \lambda-\delta\}$; this effectively eliminates the oscillatory term. Let us suppose that $c_{-}$and $e$ are cut off smoothly between $r=0$ and $r=1$ so that they are defined on the whole real line. The following lemma completes the proof.

Lemma 5. Suppose $c_{-}$is a smooth function on $\mathbb{R}$ of polynomial growth, satisfying (38), and with scattering wavefront set contained in $\{\tau \leq 2 \lambda-\delta\}$ for some $\delta>0$. If $e(r) \rightarrow e(\infty)$ as $r \rightarrow \infty$, then $2 i \lambda c_{-}(r) \rightarrow e(\infty)$ as $r \rightarrow \infty$.

Proof. Subtracting $e(\infty)$ from both $e$ and $2 i \lambda c_{-}$, we may suppose that $e(\infty)=0$. Taking the Fourier transform, we get

$$
i(\xi+2 \lambda) \hat{c}_{-}(\xi)=\hat{e}(\xi) .
$$

Since $\tau=2 \lambda$ is not in ${ }^{s c} \mathrm{WF}\left(c_{-}\right)$, by (51) the Fourier transform $\hat{c}_{-}$is smooth near $\xi=-2 \lambda$. Therefore, $\hat{e}(-2 \lambda)=0$. Let $\phi(\xi)$ be a smooth function with support contained in $[-2 \lambda-\delta,-2 \lambda+\delta / 2]$ and with $\phi=1$ in a neighbourhood of $-2 \lambda$. Then $\hat{c}_{-}$has the form

$$
\hat{c}_{-}(\xi)=c \delta(-2 \lambda)-i \frac{\hat{e} \phi}{\xi+2 \lambda}-i \frac{\hat{e}(1-\phi)}{\xi+2 \lambda} .
$$


But $\hat{c}_{-}$is smooth at $-2 \lambda$, so $c=0$. Consider the inverse Fourier transform of the two remaining terms. The first is Schwartz, since $\hat{e}$ is smooth near $-2 \lambda$ and zero at $-2 \lambda$ and the second takes the form

$$
e * k, \quad k=\mathcal{F}^{-1}\left(\frac{-i(1-\phi(\xi))}{\xi+2 \lambda}\right) .
$$

Since $e$ goes to zero at infinity, by assumption, and $k$ is $L^{1}$, this goes to zero as $r \rightarrow \infty$, as required.

We have now shown that $\lim _{r \rightarrow \infty} b_{-}(\psi, r)$ exists and equals $(2 i \lambda)^{-1} \int V u_{\omega, \lambda} \Psi$. Applying Plancherel's theorem, this is equal to

$$
\frac{(2 \pi)^{\frac{D+1}{2}}}{2 i \lambda} \lambda^{-\frac{D-1}{2}} e^{-i \pi \frac{D-1}{4}} \lim _{r \rightarrow \infty} \int T(-k,-\lambda \omega)\left(\psi(-\hat{k}) \delta(|k|-\lambda) * \hat{\chi}_{B(r, 0)}\right) d k .
$$

With $\Gamma$ defined as in step 2, note that

$$
\psi(-k) \delta(|k|-\lambda) * \hat{\chi}_{B(r, 0)} \rightarrow \psi(-k) \delta(|k|-\lambda) \text { in } \mathcal{D}^{\prime}(\Gamma) \text { as } r \rightarrow \infty .
$$

As in step 2, this implies that the limit is

$$
\left(\left(\frac{2 \pi}{\lambda}\right)^{\frac{D-1}{2}} e^{i \pi \frac{D-1}{4}}\right) \frac{i^{-D} \pi}{\lambda} \int_{|k|=\lambda} T(k,-\lambda \omega) \psi(k) d \sigma(k) .
$$

To relate this to our generalized eigenfunction $u_{g, \theta}$ we must show

Lemma 6. The limit (29) is uniform for $\omega$ in a compact subset $K$ of $S^{D-1} \backslash C$.

Proof. The limit (29) is uniform in $\omega$ if and only if the limit $b_{-}(\psi, r)$ as $r \rightarrow \infty$ is uniform, which in turn is uniform in $\omega$ if and only if the limit $c_{-}(\psi, r)$ is uniform. If we inspect the proof of Lemma 5 we see that this is true provided (a) the function $e(r)-e(\infty)$ converges to zero uniformly in $\omega$, and (b) $\hat{e}(\xi)$ and its derivative have uniform bounds in a neighbourhood of $\xi=-2 \lambda$. So consider equation (37) for $c_{-}$. It is not difficult to check from [4] that the inclusion of $u_{\omega, \lambda}$ in $\langle z\rangle^{D / 2+1+\epsilon} H^{k}$ is continuous for every $k$. This shows (a) holds. To show (b), first note that by taking enough terms in the sum (35) for $c_{-}$, the $O\left(r^{-1}\right)$ can be replaced by $O\left(r^{-l}\right)$ for any $l$, and is uniform in $\omega$ since $u_{\omega, \lambda}^{\prime}$ is uniformly in $\langle z\rangle^{D / 2+1+\epsilon} H^{k}$, so for suitable $l$, the Fourier transform of this term has uniformly bounded first and second derivatives.

As for the boundary integral in (36), it is enough to show that the Fourier transforms of $e^{i \lambda r} \int_{|z|=r} u_{\omega, \lambda}^{\prime} \Psi$ and $e^{i \lambda r} \int_{|z|=r} u_{\omega, \lambda}^{\prime} \partial_{\nu} \Psi$ are uniformly smooth at $\xi=$ $-\lambda$. Consider the first of these integrals (the second is similar). Let $B_{n}$ be the $B$ operator of Gérard, Isozaki and Skibsted in $\mathbb{R}^{n}$. Note that any function of $B$ acts 'raywise' on $\mathbb{R}^{D}$, and, if we denote the $B$ operator on $\mathbb{R}^{D}$ by $B_{D}$ and on $\mathbb{R}^{+}$by $B_{1}$, we have

$$
B_{D}(v(z))=\left(B_{1} \otimes \operatorname{Id}\right)\left(r^{(D-1) / 2} v(r \hat{z})\right)
$$

where on the right hand side, we have used the isomorphism from $L^{2}\left(\mathbb{R}^{D}\right)$ to $L^{2}\left(\mathbb{R}^{+}\right) \otimes L^{2}\left(S^{D-1}\right)$ given by $v \mapsto r^{(D-1) / 2} v(r \hat{z})$. Therefore,

$$
g\left(B_{D}\right)(v(z))=\left(g\left(B_{1}\right) \otimes \operatorname{Id}\right)\left(r^{(D-1) / 2} v(r \hat{z})\right)
$$

The one dimensional operator $B_{1}$ has the advantage that it is elliptic except at the origin, so by [4], if $\rho(r)$ is a function which vanishes on $(-\infty, 1)$ and is identically equal to one on $(2, \infty)$, and if $g$ is a symbol, then $\rho(r) g\left(B_{1}\right) \rho(r)$ is a scattering pseudodifferential operator with boundary symbol $g(-\tau)$, and it is microlocally of order $-\infty$ when $-\tau$ is away from the support of $g$. 
So consider $F_{+}\left(B_{1}\right)$ and $\tilde{F}_{-}\left(B_{1}\right)=\mathrm{Id}-F_{+}\left(B_{1}\right)$ with $F_{+}$as in step 1 . Then, $\rho F_{+}\left(B_{1}\right) \rho$ is microlocally of order $-\infty$ near $\tau=\lambda$, so for any distribution $u, \tau=\lambda$ is not in ${ }^{s c} \mathrm{WF}\left(F_{+}\left(B_{1}\right) u\right)$, or equivalently, the Fourier transform of $F_{+}\left(B_{1}\right) u$ is smooth near $\xi=-\lambda$. Since the $u_{\omega, \lambda}^{\prime}$ are uniformly in $\langle z\rangle^{D / 2+1+\epsilon} H^{k}, \int_{|z|=r} e^{i \lambda r} u_{\omega, \lambda}^{\prime} \Psi$ is uniformly in a weighted $L^{2}$ space as a function of $r$, so the Fourier transform of

$$
F_{+}\left(B_{1}\right) \int_{|z|=r} e^{i \lambda r} u_{\omega, \lambda}^{\prime} \Psi
$$

has uniformly bounded derivatives of all orders near $\xi=\lambda$.

As for $\tilde{F}_{-}\left(B_{1}\right)$, the estimates of Gérard, Isozaki and Skibsted (Theorem 2.10 of [2]) show that $\tilde{F}_{-}\left(B_{D}\right)\left(u_{\omega, \lambda}^{\prime}\right)$ is uniformly in $\langle z\rangle^{-\infty} L^{2}$. Multiplication by the function $r^{(D-1) / 2} e^{i \lambda r} \Psi$ is a pseudodifferential operator $S$ of order $(0,-(D-1) / 2)$, so by Lemma 10 in appendix [B, $\tilde{F}_{-}\left(B_{D}\right)\left(S u_{\omega, \lambda}^{\prime}\right)$ is also uniformly in $\langle z\rangle^{-\infty} L^{2}$. Therefore the Fourier transform has uniformly bounded derivatives of all orders near $\xi=-\lambda$. This completes the proof of (b).

We see from this that (28) has limit

$$
\frac{i^{-D} \pi}{\lambda} \int T(\lambda \theta,-\lambda \omega) g(\omega) d \omega .
$$

Combining this with (27) we find that the kernel of $\tilde{S}(\lambda)(\theta, \omega)$ is

$$
i^{-D+1} \delta(\theta+\omega)+\frac{i^{-D} \pi}{\lambda} T(\lambda \theta,-\lambda \omega),
$$

so we have established (25). This completes the proof of the theorem.

\section{Three-Body Plane WAVES}

In this section we shall relate the three-body plane waves constructed in 3 to those considered here. This shows that the analytic scattering matrix has the properties given in that paper.

Proposition 7. The three-body plane waves $U_{l, \omega}$ constructed in [3] agree with those constructed in [4] (and therefore, by step 1 of the proof above, also agree with the plane waves of Herbst-Skibsted).

Proof. Applying the uniqueness part of Theorem 14 of [4] to $u-u_{\omega, \lambda}$ it is sufficient to show that

$$
F_{-}(B)\left(u-e^{-i \lambda z \cdot \omega}\right) \in\langle z\rangle^{-1} L^{2},
$$

where $F_{-}(t)$ is supported on $(-\infty,-\lambda+\delta)$ and equal to one on $(-\infty,-\lambda+\delta / 2)$ for $\delta>0$ sufficiently small, and $u=U_{\omega, \theta}$ denotes the plane wave of $[3]$.

Recall that a parametrix, up to Schwartz error, for the plane wave $u$ was constructed in four different regions; let us write $u=e^{-i \lambda z \cdot \omega}+u_{1}+u_{2}+u_{3}+u_{4}+w$. Here $w$ is the final correction term given by applying the outgoing resolvent to the Schwartz error $\left(H-\lambda^{2}\right)\left(e^{-i \lambda z \cdot \omega}+u_{1}+u_{2}+u_{3}+u_{4}\right)$. The first and third regions are away from the cluster set $C$, and consequently, there is no difficulty in applying the methods of [4] (i.e., replacing $F_{-}(B)$ by the scattering pseudodifferential operator $\left.\phi^{\prime}(\Delta) F_{-}(B) \phi(\Delta)\right)$ to obtain (39) for $u_{1}+u_{3}$. As for $w$, Theorem 14 of [4] immediately gives (39). And the term $u_{4}$ is not difficult either, since it is given by a sum of terms of the form $P R_{i}\left(\lambda^{2}+i 0\right)\left(V u_{3}\right)$, where $R_{i}$ is the resolvent for the operator $\Delta+V_{i}$ on $\mathbb{R}^{D}$ and $P$ is convolution with $\hat{\rho}$ where $\rho$ is a smooth function 
with $1-\rho$ compactly supported. The methods from the proof of Theorem 14 of 4 apply to $F_{-}(B)\left(V u_{3}\right)$ since ${ }^{s c} \mathrm{WF}\left(u_{3}\right) \subset\left\{\tau<\lambda-\delta, \tau^{2}+|\mu|^{2}=\lambda^{2}\right\}$ for some $\delta>0$, and $P$ is a scattering pseudodifferential operator of order $(0,0)$, so by Lemma 10 it does not disturb the estimate. Therefore the only term remaining is $u_{2}$.

Since $u_{2}$ has an explicit integral expression as a 'paired Legendrian distribution', we will use this to show that $F_{-}(B) u_{2} \in\langle z\rangle^{-l} L^{2}$ for all $l$. As noted in the previous section,

$$
F_{-}\left(B_{D}\right)(v(z))=\left(F_{-}\left(B_{1}\right) \otimes \mathrm{Id}\right)\left(r^{(D-1) / 2} v(r \hat{z})\right),
$$

and if $\rho$ is a function vanishing near the origin, then $\rho F_{-}(B) \rho$ is a scattering pseudodifferential operator with boundary symbol $F_{-}(-\tau)$. It is more convenient to conjugate by the Fourier transform; if $\xi$ denotes the Fourier transform variable, then the interior symbol of the conjugation is $F_{-}(\xi)$. Therefore, it is enough to show that there is a function $b(\xi)$ equal to one on the support of $F_{-}(\xi)$ such that $\left.b(\xi) \widehat{\rho u_{2}(\hat{z} r}\right)(\xi)$ is a Schwartz function of $\xi$.

As a reduction, observe that by Lemma 10 it is enough to prove this for the function $v=r^{-k} u_{2}$, for any $k$. So we need to consider $\rho\left(\widehat{r) r^{-k}} u_{2}(\xi) b(\xi)\right.$. By [3], this has an local expression near a cluster set $C_{i}$, defined by $\left\{z^{\prime} / r=0\right\}, z=\left(z^{\prime}, z^{\prime \prime}\right)$, of the form

$$
\gamma(\xi) \equiv b(\xi) \int_{s \geq 0} d \zeta d t d \hat{y}^{\prime \prime} d s d r e^{-i r \xi} e^{i r \Phi\left(\hat{z}, \zeta, \hat{y}^{\prime \prime}, t, s\right)} r^{-k} \rho(r) a\left(\hat{z}, \zeta, \hat{y}^{\prime \prime}, t, s, r\right),
$$

where $\Phi$ is the function

$$
\Phi\left(\hat{z}, \zeta, \hat{y}^{\prime \prime}, t, s\right)=\hat{z} \cdot \zeta+t\left(\phi\left(0, \hat{y}^{\prime \prime}\right)-\zeta^{\prime \prime} \cdot \hat{y}^{\prime \prime}\right)-s\left(|\zeta|^{2}-\left(\zeta^{\prime \prime} \cdot \hat{y}^{\prime \prime}\right)^{2}+\phi\left(0, \hat{y}^{\prime \prime}\right)^{2}-\lambda^{2}\right),
$$

$\phi$ is a smooth function taking values in $(-\lambda+2 \delta, \lambda)$ and $a$ is smooth in all variables, symbolic in $r$ and Schwartz in $\zeta^{\prime}$, and is supported in $|s| \leq \epsilon, 0 \leq t \leq 2,\left|\zeta^{\prime \prime}\right| \leq 2$. We are free to choose the integer $k$ so we may choose it sufficiently large that the integral is absolutely convergent.

We need to show that the operations of (a) differentiation in $\xi$, (b) multiplication by powers of $\xi$ and (c) differentiation in $\hat{z}$ applied to $\gamma(\xi)$ give a uniformly bounded integral. Applying derivatives in $\xi$ to $\gamma$ brings down factors of $r$ in the integrand. We need to be able to get rid of powers of $r$ by integrating by parts in the other variables. To show this we prove the following lemma.

Lemma 8. Assume that $\operatorname{supp} b(\xi) \subset(-\infty, \lambda+\delta)$. Then on the support of $b \cdot a \cdot \rho$, the following estimates hold for some $c>0$ :

$$
|\Phi-\xi|^{2}+\left|d_{\zeta, \hat{y}^{\prime \prime}, t, s} \Phi\right|^{2} \geq c .
$$

If $\tilde{\Phi}$ is the restriction of $\Phi$ to $s=0$, then we have

$$
|\tilde{\Phi}-\xi|^{2}+\left|d_{\zeta, \hat{y}^{\prime \prime}, t} \tilde{\Phi}\right|^{2} \geq c\left\langle\zeta^{\prime}\right\rangle^{-2} .
$$

Finally, we have

$$
\left|d_{\hat{z}} \Phi\right|^{2}+\left|d_{\hat{z}} \tilde{\Phi}\right|^{2} \leq c^{-1}\left\langle\zeta^{\prime}\right\rangle^{2} .
$$

Proof. Since $\tau<\lambda-2 \delta$ on the Legendrian $L=\left\{\left(\hat{z}, \tau=-\Phi, \mu=d_{\hat{z}} \Phi\right) \mid d_{\zeta, \hat{y}^{\prime \prime}, t, s} \Phi=\right.$ $0\}$ defined by $\Phi$, and $\xi<-\lambda+\delta$ on the support of $b$, we have (41) on any compact 
subset of the support of $a$. So we only need to check (41) as $\left|\zeta^{\prime}\right| \rightarrow \infty$. But then

$$
|\Phi-\xi|^{2}+\left|d_{\zeta, \hat{y}^{\prime \prime}, t, s} \Phi\right|^{2}=(1-s)\left|\zeta^{\prime}\right|^{4}+O\left(\left|\zeta^{\prime}\right|^{3}\right)
$$

on $\operatorname{supp} b \cdot a \cdot \rho$, so (41) holds everywhere.

To prove (42), we use the inequality $(a+b+c+d)^{2} \geq a^{2} / 4-b^{2}-c^{2}-d^{2}$ to derive

$$
\begin{gathered}
|\tilde{\Phi}-\xi|^{2}=\left|\hat{z} \cdot \zeta+t \phi\left(\hat{y}^{\prime \prime}\right)-t \hat{y}^{\prime \prime} \cdot \zeta^{\prime \prime}-\xi\right|^{2} \\
\geq \frac{1}{4}\left|\phi\left(\hat{y}^{\prime \prime}\right)^{2}-\xi\right|^{2}-\left|\hat{z}^{\prime} \cdot \zeta^{\prime}\right|^{2}-\left|(1-t) \phi\left(\hat{y}^{\prime \prime}\right)\right|^{2}-\left|\left(\hat{z}^{\prime \prime}-t \hat{y}^{\prime \prime}\right) \cdot \zeta^{\prime \prime}\right|^{2} \\
\geq c \delta^{2}-\left|\hat{z}^{\prime} \cdot \zeta^{\prime}\right|^{2}-\lambda^{2}(1-t)^{2}-\left|\zeta^{\prime \prime} \cdot d_{\zeta^{\prime \prime}} \tilde{\Phi}\right|^{2} \\
\geq c \delta^{2}-\left|\hat{z}^{\prime} \cdot \zeta^{\prime}\right|^{2}-\lambda^{2}\left|d_{\zeta^{\prime \prime}} \tilde{\Phi}\right|^{2}-4\left|d_{\zeta^{\prime \prime}} \tilde{\Phi}\right|^{2} .
\end{gathered}
$$

Thus, if we add to the right hand side

$$
\left(4+\lambda^{2}\right)\left\langle\zeta^{\prime}\right\rangle^{2}\left|d_{\zeta} \tilde{\Phi}\right|^{2}=\left(4+\lambda^{2}\right)\left\langle\zeta^{\prime}\right\rangle^{2}\left(\left|\hat{z}^{\prime}\right|^{2}+\left|d_{\zeta^{\prime \prime}} \tilde{\Phi}\right|^{2}\right)
$$

then the result is at least $c \delta^{2}$, proving (42).

The estimate (43) is obvious.

The estimate allows us to integrate by parts repeatedly in $r, \zeta, \hat{y}^{\prime \prime}, t, s$ to remove arbitrarily many powers of $r$. In doing so we get boundary terms at $s=0$ leading to a similar integral involving $\tilde{\Phi}$, and then we use (42) to integrate by parts (the factor $\left\langle\zeta^{\prime}\right\rangle^{-2}$ causes no problems since $a$ is Schwartz in $\left.\zeta^{\prime}\right)$.

If we include a factor $\xi$ in the integral, this may be written $(\Phi-\xi)+\Phi$. The $\Phi-\xi$ is equivalent to taking an $r$ derivative of the exponential, which may be integrated by parts, while multiplying $a$ by $\Phi$ is harmless since $a$ is Schwartz in $\zeta^{\prime}$. Thus we can bound the integral after multiplying by any power of $\xi$.

Finally, taking $\hat{z}$ derivatives at worst brings down factors of $r d_{\hat{z}} \Phi$. As we have seen powers of $r$ can be removed via integration by parts, and multiplying $a$ by $d_{\hat{z}} \Phi$ is harmless by 43 . We conclude that $\partial_{\hat{z}}^{\alpha}(\xi)^{l} \partial_{\xi}^{m} \gamma(\xi)$ is bounded uniformly in $\hat{z}$, completing the proof of the proposition.

\section{PushForward TheOrem}

In this section we state and prove the pushforward theorem for the scattering wavefront set.

Let $f: \tilde{X} \rightarrow X$ be a smooth map between manifolds with boundary, such that $f$ has surjective differential, and such that if $x$ is a boundary defining function for $X$ (that is, $x \geq 0$ on $X, x=0$ on $\partial X$ and $d x \neq 0$ on $\partial X$ ) then $f^{*} x$ is a boundary defining function for $\tilde{X}$. Then in suitable local coordinates on $\tilde{X}$ and $X$, the map $f$ takes the form

$$
(x, y, w) \mapsto(x, y)
$$

near the boundary, or

$$
(z, w) \mapsto z
$$

in the interior of $\tilde{X}$. Thus, $f$ is locally identical to the projection $B^{n} \times T^{k} \rightarrow B^{n}$, where $T^{k}$ is the $k$-torus.

The map $f$ pushes forward tempered distributional densities on $\tilde{X}$ (objects dual to smooth functions with all derivatives vanishing at the boundary) to tempered distributional densities on $X$, via 'integration along the fibres'. It is important in 
many contexts to have bounds on the scattering wavefront set of the pushforward of $u$, denoted $f_{*} u$, in terms of the scattering wavefront set of $u$. This may be described in terms of a relation between $C(\tilde{X})$ and $C(X)$ induced by $f$. The relation comes from the mapping from $T_{f(p)}^{*}(X)$ to $T_{p}^{*}(\tilde{X})$ induced by $f$. Here $C(X)$ is the boundary of the fibrewise compactification of the scattering cotangent bundle (see [10, section 5, or appendix $\mathrm{A}$ of this paper in the case that $X$ is the radial compactification $\overline{\mathbb{R}^{D}}$ of $\mathbb{R}^{N}$ ). It is easiest, and most useful, to describe the map in local coordinates based on the standard forms (44) and (45). There are three disjoint regions of $C(X)$, (i) above the interior of $X$, (ii) above the boundary of $X$ and not at fibre-infinity or (iii) the corner, i.e. above the boundary of $X$ and at fibre-infinity. The relation only associates points in $C(X)$ to points in the corresponding region of $C(\tilde{X})$. We use coordinates $\zeta$ and $\lambda$ dual to $z$ and $w$, and use $\tau, \mu$ and $\xi$ for the coordinates on the scattering cotangent bundle given by the coefficients of $d x / x^{2}, d y / x$ and $d w / x$ (so $\xi=x \lambda$ ). The meaning of the $\tau$ and $\mu$ coordinates when $X=\overline{\mathbb{R}^{D}}$ is explained in the appendix.

In the first region, the relation is

$$
(z ; \zeta) \sim(\tilde{z}, w ; \tilde{\zeta}, \lambda) \text { iff } \tilde{z}=z, \tilde{\zeta}=\zeta, \lambda=0,
$$

in the second region, the relation is

$$
(0, y ; \tau, \mu) \sim(0, \tilde{y}, w ; \tilde{\tau}, \tilde{\mu}, \xi) \text { iff } \tilde{y}=y, \tilde{\tau}=\tau, \tilde{\mu}=\mu, \xi=0,
$$

and in the third region, the relation is

$$
(0, y ; \infty(\tau, \mu)) \sim(0, \tilde{y}, w ; \infty(\tilde{\tau}, \tilde{\mu}, \xi)) \text { iff } \tilde{y}=y,(\tilde{\tau}, \tilde{\mu})=a(\tau, \mu), a>0, \xi=0 .
$$

Here $\lambda$ is the cotangent variable dual to $w$ and $\xi$ is the rescaled variable $x \lambda$.

Theorem 9 (Pushforward Theorem). If $u$ is a tempered distributional density on $\tilde{X}$, then $f_{*} u$ satisfies

$$
{ }^{s c} W F\left(f_{*} u\right) \subset\left\{q \in C(X) \mid \exists \tilde{q} \in{ }^{s c} W F(u) \text { with } \tilde{q} \sim q\right\} .
$$

Proof. Suppose that $q \in C(X)$ is not a element of the set in the right hand side of (49). We will show that it is not in ${ }^{s c} \mathrm{WF}\left(f_{*} u\right)$.

Consider the three regions described above in which $q$ may lie. Let us treat the case (iii) fully, as it is the most difficult case, and comment briefly on the proof for the other two cases. In each case, the structure of the proof is the same. We need to find a scattering pseudodifferential operator $A$ on $X$ which is elliptic at $q$ and such that $A\left(f_{*} u\right)$ is in $\dot{C}^{\infty}(X)$. We do this by constructing $A$ and a corresponding pseudodifferential operator $\tilde{A}$ on $\tilde{X}$ such that ${ }^{s c} \mathrm{WF}^{\prime}(\tilde{A})$ is disjoint from ${ }^{s c} \mathrm{WF}(u)$, and such that

$$
f_{*}(\tilde{A} u)=A\left(f_{*} u\right)+R\left(f_{*} u\right), \quad R \in \Psi^{-\infty, \infty}(X) .
$$

Then by the pseudodifferential calculus, $A\left(f_{*} u\right)$ is in $\dot{C}^{\infty}(X)$, as required.

Localizing on $\tilde{X}$ with a partition of unity, we may assume $u$ has support in a small open set, in which case the situation is identical with that of the model situation where $f$ is the projection $B^{n} \times T^{k} \rightarrow B^{n}$. Thus we need only prove the result in this case.

Let us use coordinates $z$ on $B^{n}, z \in \mathbb{R}^{n}$, and $w$ on $T^{k}$. Smooth coordinates near the projection of $q$ are $(x, y)$ where $x=1 /|z|$ and $y$ are local coordinates on $S^{n-1}$. However, we will use $z$ even near the boundary of $\overline{\mathbb{R}^{n}}$ since it is convenient 
for writing down the kernels of scattering pseudodifferential operators. Let $\zeta$ be cotangent coordinates dual to $z, \lambda$ cotangent coordinates dual to $w$ and $\xi$ the rescaled coordinate $x \lambda$.

By hypothesis the sets ${ }^{s c} \mathrm{WF}(u)$ and $\{\tilde{q} \mid \tilde{q} \sim q\}$ are disjoint closed sets, so in view of (46) - 48) we can find an open set $U \subset C(X)$ containing $q$, and $\delta>0$ such that $(z, \zeta, w, \xi) \notin{ }^{s c} \mathrm{WF}(u)$ if $(z, \zeta) \in U$ and $|\xi| \leq \delta\langle\zeta\rangle$. Choose a pseudodifferential operator $A$ of order $(0,0)$ on $B^{N}$ with ${ }^{s c} \mathrm{WF}^{\prime}(A) \subset U$. Let $a(z, \zeta)$ be the leftreduced symbol of $A$. Also choose $\phi \in \mathcal{S}$ such that $\phi(0)=1$ and $\phi(\xi)$ is supported in $|\xi| \leq \delta$. Finally choose $\psi \in C_{c}^{\infty}(\mathbb{R})$ so that $\psi(t)=1$ if $|t|<1 / 4$ and $\psi(t)=0$ if $|t|>1 / 2$. Consider the operator acting on densities on $B^{n} \times T^{k}$ with kernel

$$
\begin{gathered}
\tilde{A}\left(z, z^{\prime}, w, w^{\prime}\right)|d z d w| \\
A\left(z, z^{\prime}, w, w^{\prime}\right)=\frac{\psi\left(\left|w-w^{\prime}\right|\right)}{(2 \pi)^{n+k} x^{k}} \int e^{i\left(z-z^{\prime}\right) \cdot \zeta} e^{i\left(\left(w-w^{\prime}\right) / x\right) \cdot \xi} a(z, \zeta) \phi\left(\frac{\xi}{\langle\zeta\rangle}\right) d \xi d \zeta .
\end{gathered}
$$

Since $a(z, \zeta) \phi(\xi /\langle\zeta\rangle)$ is a symbol in $(\zeta, \xi)$, this is a pseudodifferential operator on $B^{N} \times T^{k}$. Computing $f_{*}(\tilde{A} u)$, we find that

$$
\begin{gathered}
f_{*}(\tilde{A} u)=\int d w \int A\left(z, z^{\prime}, w, w^{\prime}\right) f\left(z^{\prime}, w^{\prime}\right) d z^{\prime} d w^{\prime}|d z| \\
=\left(\int d w^{\prime \prime} \psi\left(\left|w^{\prime \prime}\right|\right) \hat{\phi}\left(\frac{w^{\prime \prime}\langle\zeta\rangle}{x}\right)\langle\zeta\rangle^{k} x^{-k}\right) \int e^{i\left(z-z^{\prime}\right) \cdot \zeta} a(z, \zeta) f\left(w^{\prime}, z^{\prime}\right) \frac{d w^{\prime} d z^{\prime} d \zeta}{(2 \pi)^{n}}|d z|
\end{gathered}
$$

where $w^{\prime \prime}=w-w^{\prime}$. Let $v=w^{\prime \prime}\langle\zeta\rangle / x$, then the first factor in large parentheses is

$$
\int d v \hat{\phi}(v) \psi\left(\frac{v x}{\langle\zeta\rangle}\right) d v=1+r\left(\frac{x}{\langle\zeta\rangle}\right)
$$

with $r \in \mathcal{S}$. Therefore $f_{*}(\tilde{A} u)$ is equal to

$$
\int\left(1+r\left(\frac{x}{\langle\zeta\rangle}\right)\right) e^{i\left(z-z^{\prime}\right) \cdot \zeta} a(z, \zeta) f\left(w^{\prime}, z^{\prime}\right) \frac{d w^{\prime} d z^{\prime} d \zeta}{(2 \pi)^{n}}|d z|=A\left(f_{*} u\right)+R\left(f_{*} u\right)
$$

where $R$ is of order $(-\infty, \infty)$. This completes the proof in case (iii).

In case (ii), we find an open set $U \subset C(X)$ containing $q$, and $\delta>0$ such that $(z, \zeta, w, \xi) \notin{ }^{s c} \mathrm{WF}(u)$ if $(z, \zeta) \in U$ and $|\xi| \leq \delta$. Then we choose a pseudodifferential operator $A$ of order $(-\infty, 0)$ on $B^{N}$ with ${ }^{s c} \mathrm{WF}^{\prime}(A) \subset U$ and choose $\phi$ as before. Then $\tilde{A}$ is defined by

$$
\begin{gathered}
\tilde{A}\left(z, z^{\prime}, w, w^{\prime}\right)|d z d w| \\
A\left(z, z^{\prime}, w, w^{\prime}\right)=\frac{\psi\left(\left|w-w^{\prime}\right|\right)}{(2 \pi)^{n+k} x^{k}} \int e^{i\left(z-z^{\prime}\right) \cdot \zeta} e^{i\left(\left(w-w^{\prime}\right) / x\right) \cdot \xi} a(z, \zeta) \phi(\xi) d \xi d \zeta .
\end{gathered}
$$

In case (i) a similar modification works, or alternatively this is just the standard pushforward result for the wavefront set ([7], Theorem 8.2.12).

\section{Appendix A. The scattering Wavefront Set}

Here we collect a few fundamental facts about the scattering wavefront set. For more detail see [10] or [12].

The scattering wavefront set was introduced by Melrose 10] in the context of manifolds with boundary $X$ and 'scattering metrics' (a class of complete metrics on $X$ which are asymptotically flat at the boundary). In this paper, we mostly 
consider the case where $X$ is the radial compactification of $\mathbb{R}^{D}$ which we will identify with $B^{D}$. The scattering wavefront set incorporates both interior singularities and singularities at the boundary, but in this paper we are interested mostly in the part of the scattering wavefront set at the boundary (infinity) since we are dealing with generalized eigenfunctions which have no interior singularities.

The scattering wavefront set is defined in terms of a calculus of pseudodifferential operators, the scattering pseudodifferential operators. On $\mathbb{R}^{D}$, a linear operator $A$ is a (classical) scattering pseudodifferential operator if its Schwartz kernel can be expressed as an oscillatory integral

$$
A\left(z, z^{\prime}\right)=(2 \pi)^{-D} \int e^{i\left(z-z^{\prime}\right) \cdot \zeta} a(z, \zeta) d \zeta
$$

where $a$ is $\langle z\rangle^{-l}\langle\zeta\rangle^{m}$ times a smooth function $b$ on $\overline{\mathbb{R}^{D}} \times \overline{\mathbb{R}^{D}}$. That is, $b$ is smooth in the differentiable structure on $T^{*}\left(\mathbb{R}^{D}\right)$ obtained by compactifying both $\mathbb{R}_{z}^{D}$ and $\mathbb{R}_{\zeta}^{D}$ factors into an $D$-ball $B^{D}$. Then $a$ is said to be a symbol of order $(m, l)$. This condition can be alternatively expressed by saying that $a$ is a classical symbol in both $z$ and $\zeta$, with the symbol estimates in $\zeta$ satisfied uniformly with respect to $z$ and conversely. Scattering pseudodifferential operators extend to well-defined maps on tempered distributions.

Such operators have a principal 'boundary symbol' defined at spatial infinity as well as the usual principal symbol defined in the interior. If $A$ is a scattering pseudodifferential operator with symbol $a$ of order $(m, 0)$, the boundary symbol of $A$ is defined to be

$$
\sigma(A)(\hat{z}, \zeta)=\lim _{r \rightarrow \infty} a(r \hat{z}, \zeta) .
$$

This limit exists by our assumptions on $a$. Thus the boundary symbol is a smooth function on the set $K=S_{\hat{z}}^{D-1} \times \mathbb{R}_{\zeta}^{D}$. It is not homogeneous in $\zeta$ in general, though it is symbolic of order $m$ in $\zeta$.

The joint principal symbol of $A$ is the pair consisting of the boundary symbol and the usual interior principal symbol which, in the classical case, can be taken to be given by the leading asymptotic of $a(z, \zeta)$ as $|\zeta| \rightarrow \infty$. These data are not independent, but satisfy a compatibility condition which is captured by the function $j(A)$ defined on $C\left(\overline{\mathbb{R}^{D}}\right)$, the boundary of the manifold with corners $\overline{\mathbb{R}^{D}} \times \overline{\mathbb{R}^{D}}$. The set $C\left(\overline{\mathbb{R}^{D}}\right)$ consists of the union of $\bar{K}$, the compactification of $K$, and $\overline{\mathbb{R}^{D}} \times S_{\hat{\zeta}}^{N-1}$ (the cosphere bundle of $\mathbb{R}^{D}$, compactified at infinity) along their common boundary. The function $j(A)$ is defined by

$$
j(A)=\langle z\rangle^{l}\langle\zeta\rangle^{-m} a(z, \zeta)\left\lceil C\left(\overline{\mathbb{R}^{D}}\right)\right.
$$

and combines the information in the interior and boundary symbols. If $A$ is of order $(m, l)$ and $q \in C\left(\overline{\mathbb{R}^{D}}\right)$ is such that $j(A)(q) \neq 0$ then $q$ is said to be an elliptic point for $A$.

For a general manifold $X$ with boundary $y$, the class of scattering pseudodifferential operators can be defined from the calculus on $\mathbb{R}^{D}$ via localization (see [10], section 4). In this case, the boundary symbol is defined on the set $K(X)$ which can be identified (non-canonically) with $T^{*} Y \times \mathbb{R}$, and the joint symbol is defined on $C(X)$ which can be identified (non-canonically) with the boundary of $\overline{T^{*} X}$, the fibrewise radial compactification of the cotangent bundle of $X$. As before this consists of the union of two manifolds along their common boundary, an interior part which is diffeomorphic to the cosphere bundle of $X$ and a part 'at infinity' which 
is a compactified version of $K(X)$. A more intrinsic description of $C(X)$ requires discussion of the scattering cotangent bundle; see [10] for more details.

If $u$ is a tempered distribution on $\mathbb{R}^{D}$, the scattering wavefront set of $u$ is the closed subset ${ }^{s c} \mathrm{WF}(u)$ of $C\left(\overline{\mathbb{R}^{D}}\right)$ whose complement is given by

$$
{ }^{s c} \mathrm{WF}(u)^{\complement}=\left\{q \in C\left(\overline{\mathbb{R}^{D}}\right) \mid \exists A \text { such that } A u \text { is Schwartz and } q \text { is elliptic for } A\right\} .
$$

The definition works in the general scattering metric setting when Schwartz space and the space of tempered distributions are replaced by $\dot{C}^{\infty}(X)$, the space of functions all of whose derivatives vanish at $\partial X$, and its dual. In this paper, we are mostly interested in $C^{\infty}$ functions whose scattering wavefront set is contained in the boundary part, $K$, of $C\left(\overline{\mathbb{R}^{D}}\right)$, for which we have coordinates $(\hat{z}, \zeta)$. For smooth functions $u$ on $\mathbb{R}^{D}$, there is an alternative definition of the scattering wavefront set as the complement of the set

$$
\left\{\left(\hat{z}_{0}, \zeta_{0}\right) \mid \exists C^{\infty} \text { function } \phi(x, \hat{z}) \text { with } \phi\left(0, \hat{z}_{0}\right) \neq 0, \widehat{\phi u}(\zeta) \text { smooth near } \zeta=\zeta_{0}\right\} \text {. }
$$

For example, the scattering wavefront sets of the functions $e^{-i z \cdot \theta}, \cos \lambda|z|$ and $V\left(z^{\prime}\right)$, for $V \in \mathcal{S}\left(\mathbb{R}^{d}\right)$, are respectively

$$
\{(\hat{z}, \zeta=-\theta)\}, \quad\{(\hat{z}, \zeta=\lambda \hat{z})\} \quad \text { and } \quad\left\{(\hat{z}, \zeta) \mid \hat{z}^{\prime}=0, \zeta^{\prime \prime}=0\right\}
$$

Here $z \in \mathbb{R}^{D}$ is decomposed as $z=\left(z^{\prime}, z^{\prime \prime}\right), z^{\prime} \in \mathbb{R}^{d}$ and $\zeta$ is decomposed similarly. Informally, the set of $\zeta$ such that $\left(\hat{z}_{0}, \zeta\right) \in{ }^{s c} \mathrm{WF}(u)$ may be thought of as the Fourier modes present in $u$ at infinity, in a small open cone containing the direction $\hat{z}_{0}$.

There is a duality between the scattering wavefront set for smooth functions on $\mathbb{R}^{D}$ and the wavefront set of their Fourier transforms: namely,

$$
(\hat{z}, \zeta) \in{ }^{s c} \mathrm{WF}(u) \text { if and only if }(\zeta,-t \hat{z}) \in \mathrm{WF}(\hat{u}) \text { for } t>0 \text {. }
$$

If at least one of $u$ and $v$ have scattering wavefront set contained in $\{|\zeta| \leq R\}$, then under multiplication, the scattering wavefront set obeys the relation

$$
{ }^{s c} \mathrm{WF}\left(u_{1} \cdot u_{2}\right) \subset\left\{(\hat{z}, \zeta) \mid \exists\left(\hat{z}, \zeta_{1}\right) \in{ }^{s c} \mathrm{WF}\left(u_{1}\right),\left(\hat{z}, \zeta-\zeta_{1}\right) \in{ }^{s c} \mathrm{WF}\left(u_{2}\right)\right\} .
$$

It is often convenient to use the coordinates $(\hat{z}, \tau, \mu)$ instead of $(\hat{z}, \zeta)$, on $K$, where $\tau \in \mathbb{R}$ and $\mu \perp \hat{z}$. These coordinates are linked by $\zeta=-\tau \hat{z}+\mu$, so $(\tau, \mu)$ are the components of $\zeta$ with respect to $\hat{z}$. Thus, $\mu$ can be thought of as the dual variables to $\hat{z}$ in $T^{*}\left(S^{D-1}\right)$, and then this identifies $K$ with $T^{*}\left(S^{D-1}\right) \times \mathbb{R}$. More generally, on a compact manifold $X$ with boundary $Y, K(X)$ can be identified (non-canonically) with $T^{*} Y \times \mathbb{R}$; the identification is non-canonical because it depends on a choice of boundary defining function $x$.

\section{Appendix B. The $B$ operator and the commutator expansion}

The $B$ operator introduced by Gérard, Isozaki and Skibsted is

$$
B=\frac{1}{2 i} \sum_{j}\left(\frac{z_{j}}{\langle z\rangle} \partial_{j}+\partial_{j} \frac{z_{j}}{\langle z\rangle}\right)
$$

In [1], [2] functions of this operator were used to derive $N$ body resolvent estimates. These estimates are also discussed and used in [4]. A typical function $F(B)$ of 
interest is of the form

$$
F \in C^{\infty}, F(t)= \begin{cases}0, & t<t_{0} \\ 1, & t>t_{1} .\end{cases}
$$

To define $f(B)$ for symbols $g$ of order $m<0$, one can use the formula of HelfferSjöstrand [5]:

$$
g(A)=\frac{1}{2 \pi} \int_{\mathbb{C}} \overline{\partial_{\lambda}} G(\lambda)(A-\lambda)^{-1} d \lambda d \bar{\lambda} .
$$

Here $G$ is an almost analytic extension of $g$, that is, a function defined on $\mathbb{C}$ such that $G(\lambda)$ agrees with $f$ on the real axis and satisfies

$$
\begin{gathered}
\operatorname{supp} G \subset\{\lambda|| \operatorname{Im} \lambda|<1+| \operatorname{Re} \lambda \mid\} ; \\
|\bar{\partial} G| \leq C_{N}|\operatorname{Im} \lambda|^{N}\langle\lambda\rangle^{m-1-N}, \quad N=0,1,2 \ldots
\end{gathered}
$$

Then one can derive the following formula for $[A, g(B)]$ for a symbol $g$ of any order:

$$
\begin{gathered}
{[A, g(B)]=\sum_{j=1}^{n-1} \frac{(-1)^{j+1}}{j !} \operatorname{ad}_{j}(A, B) g^{(j)}(B)+R_{n}, \quad n>\text { ord } g} \\
R_{n}=-\frac{1}{2 \pi} \int_{\mathbb{C}} \bar{\partial} G(\lambda)(B-\lambda)^{-1} \operatorname{ad}_{n}(A, B)(B-\lambda)^{-n} d \lambda d \bar{\lambda} .
\end{gathered}
$$

The following lemma, which is implicit in [4], is used in several places in this paper. Recall $H^{k, l}$ denotes the weighted Sobolev space $\langle z\rangle^{-l} H^{k}$.

Lemma 10. Suppose that $v \in H^{0, l}$ is a function on $\mathbb{R}^{n}$ such that $f(B) v \in H^{0, \infty}$ for all $f$ with support in an open set $O \subset \mathbb{R}$. If $g$ is a symbol supported in $O$, then for all scattering pseudodifferential operators $P$ of order $(0, k), k$ arbitrary, we have

$$
g(B) P v \in H^{0, \infty} \text {. }
$$

Proof. We write

$$
g(B) P v=P g(B) v+[g(B), P] v .
$$

The first term on the right hand side is in $H^{0, \infty}$ since $g(B) v$ is, by hypothesis, so we need to show that the commutator term is in $H^{0, \infty}$. Expanding the commutator via (57), we see that each term in the expansion lies in $H^{0, \infty}$ since $g^{(j)}$ has support in $O$ and $\operatorname{ad}_{j}(P, B)$ is a pseudodifferential operator of order $(0, k-j)$. So consider the remainder term. It is shown in 4 that $(B-\lambda)^{-1}$ is bounded on $H^{0, l}$ with operator bound $C\left(|\operatorname{Im} \lambda|^{-1}+|\operatorname{Im} \lambda|^{-|l|-1}\right)$, where $C$ is independent of $\lambda$. Using this, the bound (56) and the fact that $\operatorname{ad}_{n}(P, B)$ is a pseudodifferential operator of order $(0, k-n)$, it follows that the remainder $R_{n}$ maps $H^{0, l}$ to $H^{0, l-k+n}$ for all $l$. Since $n$ is arbitrary, we have shown that the commutator term lies in $H^{0, m}$ for all $m$.

\section{REFERENCES}

1. C. Gérard, H. Isozaki and E. Skibsted, Commutator algebra and resolvent estimates, Advanced Studies in Pure Mathematics, vol. 23, 1994. MR 95h:35154

2. C. Gérard, H. Isozaki and E. Skibsted, $N$-body resolvent estimates, J. Math. Soc. Japan 48, 1996, 135-160. MR 96j:81131

3. A. Hassell, Plane waves for the 3 body Schrödinger operator, Geom. and Funct. Anal., to appear. 
4. A. Hassell and A. Vasy, Symbolic functional calculus and $N$ body resolvent estimates, J. Funct. Anal., to appear.

5. B. Helffer, J. Sjöstrand, Equation de Schrödinger avec champ magnétique et équation de Harper, in Lecture Notes in Physics, vol. 345, H. Holder and A. Jensen (eds.), Springer, 1989. MR 91g:35078

6. I. Herbst and E. Skibsted, Free channel Fourier transform in the long range $N$-body problem, Jour. d'Anal. Math. 65, 1995, 297-332. MR 96j:81132

7. L. Hörmander, The analysis of linear partial differential operators I, Springer, second edition, 1990. MR 91m:35001a

8. H. Isozaki, A generalization of the radiation condition of Sommerfeld for $N$-body Schrödinger operators, Duke Math. J. 74, 1994, 557-584. MR 95d:81148

9. A. Jensen and H. Kitada, Fundamental solutions and eigenfunctions expansions for Schrödinger operators II: eigenfunction expansions, Math. Z. 199, 1988, 1-13. MR 90a:35176

10. R. B. Melrose, Spectral and scattering theory for the Laplacian on asymptotically Euclidean spaces, Spectral and scattering theory (M. Ikawa, editor), Marcel Dekker, 1994. MR 95k:58168

11. R. B. Melrose, Geometric scattering theory, Cambridge University Press, 1995. MR 95k:35129

12. R. B. Melrose and M. Zworski, Scattering metrics and geodesic flow at infinity, Invent. Math. 124, 1996, 389-436. MR 96k:58230

13. P. Perry, I. M. Sigal, B. Simon, Spectral analysis of N-body Schrödinger equations, Annals of Math. 114, 1981, 519-567. MR 83b:81129

14. M. Reed and B. Simon, Methods of modern mathematical physics III: Scattering theory, Academic Press, 1979. MR 80m:81085

15. I. M. Sigal and A. Soffer, The $N$-particle scattering problem: asymptotic completeness for short-range systems, Annals of Math. 126, 1987, 35-108. [MR 88m:81137]

16. A. Vasy, Structure of the resolvent for three-body potentials, Duke Math. J. 90, 1997, 379-434. MR 98k:81295

17. A. Vasy, Asymptotic expansion of generalized eigenfunctions in $N$-body scattering, J. Funct. Anal. 148, 1997, 170-184. MR 98f:81344

18. A. Vasy, Propagation of singularities in three-body scattering, M.I.T. PhD thesis, 1997.

19. A. Vasy, Scattering matrices in many-body scattering, Commun. Math. Phys. 200, 1999, 105-124. CMP 99:08

Centre for Mathematics and its Applications, Australian National University, CanBerra ACT 0200, Australia

E-mail address: hassell@maths.anu.edu.au 